\title{
New Materials Design
}

\author{
Jerry Boatz ${ }^{1}$, Mark S. Gordon ${ }^{2}$, Gregory Voth ${ }^{3}$, Sharon Hammes-Schiffer ${ }^{4}$, and \\ Ruth Pachter \\ Air Force Research Laboratory, AFRL/PRSP, Edwards AFB, CA 93524 \\ Department of Chemistry, Iowa State University, Ames, IA 50011 \\ ${ }^{3}$ Department of Chemistry and Henry Eyring Center for Theoretical Chemistry, University \\ of Utah, Salt Lake City, UT 84112 \\ ${ }^{4}$ Department of Chemistry, The Pennsylvania State University, University Park, PA 16802 \\ Air Force Research Laboratory, AFRL/MLPJ, Wright-Patterson AFB, OH 45433
}

\begin{abstract}
In this paper we report our recent results on the design of materials with controlled properties by the application of computational chemistry methods, new algorithms and scalable software.
\end{abstract}

\section{Introduction}

Developments of new materials, such as new high-energy POSS (polyhedral oligomeric silsesquioxanes), that are highly resistant to extreme environments and are therefore desirable coatings for rocket engines, nonlinear optical materials, and liquid crystals, are important in many applications of interest to the Air Force. However, the design of large and complex material systems with controlled properties is challenging. Therefore, the calculations we describe in this paper would not have been possible without scalable quantum chemistry codes such as GAMESS (General Atomic and Molecular Electronic Structure System), developed under the auspices of a DoD CHSSI (Computational High Performance Scalable Software Initiative) grant, Gaussian, and molecular dynamics codes, as well as the availability of the DoD Computation Centers through our computational challenge project.

\section{Results and Discussion}

\subsection{High-Energy Density Materials (HEDM)}

\subsubsection{Bond Dissociation Energies: A Comparative Study of Theoretical

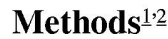

The purpose of this study is to perform a systematic comparison of several theoretical methods for computing the bond dissociation energies (BDEs) of a prototypical energetic compound. The computational methods include single-reference (MollerPlesset second order perturbation theory (MP2)[1], Gaussian-2 using MP2 (G2(MP2))[2], and coupled cluster with single and double substitutions $(\operatorname{CCSD}(\mathrm{T}))[3])$, multiconfigurational (complete active space self-consistent field

1 This work was done in collaboration with Donald L. Thompson and Dan C. Sorescu, Department of Chemistry, Oklahoma State University, Stillwater, OK 74078.

${ }^{2}$ We thank Dr. Jeffrey A. Sheehy for several stimulating discussions. 
(CASSCF)[4] and multiconfiguration quasi-degenerate perturbation theory (MCQDPT)[5]), as well as density functional theory (DFT, using Becke's three parameter hybrid exchange functional with the Lee, Yang, and Parr correlation (B3LYP)[6]) methods. The energetic compounds of interest are 1,1-diamino-2,2dinitroethylene (FOX-7) and its simpler analogues. FOX-7 was recently synthesized[7] and found to have superior impact and friction sensitivity properties relative to the well-known HMX and RDX explosives. This molecular system is an example of a "push-pull" ethylene compound, which has both electron donating $\left(\mathrm{NH}_{2}\right)$ and electron withdrawing $\left(\mathrm{NO}_{2}\right)$ groups attached to a common ethylene linkage. Such compounds are potentially stabilized through " $\pi$ " resonance delocalization and hence may possess non-negligible multiconfigurational character. Therefore, this molecule should be a good testbed for comparing DFT and single-configuration methods against multiconfigurational techniques.

The geometries of the reactants and products of the C-NH $\mathrm{N}_{2}$ and $\mathrm{C}-\mathrm{NO}_{2}$ homolytic bond dissociation reactions of FOX-7 and its analogues have been fully optimized and verified as local minima at the B3LYP, MP2 and CASSCF levels, using the 6$311 \mathrm{G}(\mathrm{d}, \mathrm{p})$ basis set.[8] CCSD(T)//MP2, MCQDPT//CASSCF, and G2(MP2) energies were also computed. The putative resonance delocalization of the " $\pi$ " electrons in FOX-7 suggests that in addition to the $(2 \mathrm{e}, 2 \mathrm{o}) \mathrm{C}-\mathrm{N} \sigma, \sigma^{*}$ space needed to describe homolytic bond cleavage, an appropriate active space for the MCSCF calculations should include the $(2 \mathrm{e}, 2 \mathrm{o}) \mathrm{C}-\mathrm{C} \pi+\pi^{*},(4 \mathrm{e}, 3 \mathrm{o}) \mathrm{NO}_{2} \pi+$ non-bonding $+\pi^{*}$, and $(2 \mathrm{e}, 1 \mathrm{o}) \mathrm{NH}_{2}$ lone pair electrons and orbitals, described in further detail elsewhere.[9] The degree of multiconfigurational character is assessed by calculation of the natural orbital occupation numbers (NOONs) of the MP2, CCSD(T), and CASSCF first order density matrices. As shown in a recent work by Gordon[10] et al., the occurrence of "non-physical" MP2 and CCSD(T) NOONs is an indication that a single configuration treatment may be inadequate. All calculations were performed using GAMESS[11], GAUSSIAN98[12], and ACESII.[13]

At the MP2 level, aminoethylene is the only closed shell compound without at least one non-physical NOON. Although some calculations are still in progress, it appears that all open-shell species except the amino and nitro radicals have multiple nonphysical NOONs at this level of theory. In contrast, none of the compounds have nonphysical NOONs at the CCSD(T) level, although several open shell CCSD(T) calculations are still in progress. This indicates that $\operatorname{CCSD}(\mathrm{T})$ is able to capture more of the non-dynamical correlation than MP2 and hence should be more reliable. The CASSCF NOONs show that the lone pair orbital on the $\mathrm{NH}_{2}$ group has little multiconfigurational character. However, the nominally unoccupied $\mathrm{NO}_{2} \pi^{*}(0.08$ 0.14 electrons) orbitals have relatively large populations, suggesting that the $\mathrm{C}-\mathrm{NO}_{2}$ BDEs may not be as well described by single reference methods as the $\mathrm{C}-\mathrm{NH}_{2}$ BDEs.

The predicted $\mathrm{C}-\mathrm{NH}_{2}$ BDEs for aminoethylene (which include zero-point energy corrections) are all within $9 \mathrm{kcal} / \mathrm{mol}$ of the experimental value of $102 \mathrm{kcal} / \mathrm{mol}[14]$ and range from 93 (MCQDPT) to 108 (MP2) kcal/mol. The B3P86/6-31+G(d,p)[15] and G2(MP2) predictions are both within about $1 \mathrm{kcal} / \mathrm{mol}$ of experiment. MP2 overestimates the BDE by $6 \mathrm{kcal} / \mathrm{mol}$ and MCQDPT underestimates it by about 9 $\mathrm{kcal} / \mathrm{mol}$, with CCSD(T) $(97.4 \mathrm{kcal} / \mathrm{mol})$ and B3LYP (96.7) yielding intemediate values. $\operatorname{CCSD}(\mathrm{T})$ is the single reference method in closest agreement with MCQDPT.

For 1-amino-2-nitroethylene, MP2 again gives the largest $\mathrm{C}-\mathrm{NH}_{2}$ BDE (126 $\mathrm{kcal} / \mathrm{mol})$ and MCQDPT the smallest $(112 \mathrm{kcal} / \mathrm{mol})$. CCSD(T) is again the singlereference method in closest agreement with MCQDPT. Although the $\mathrm{C}_{-} \mathrm{NH}_{2} \mathrm{BDE}$ 
calculations for the entire set of ethylene derivatives are incomplete, it appears that (a) MP2 consistently gives the highest $\mathrm{C}-\mathrm{NH}_{2}$ BDEs and (b) the MCQPDT BDEs are in good agreement with $\mathrm{CCSD}(\mathrm{T})$. In contrast, the DFT BDEs show no clear patterns of behavior. For nitroethylene, the $\mathrm{C}-\mathrm{NO}_{2}$ BDEs range from $59.5 \mathrm{kcal} / \mathrm{mol}$ (MCQDPT) to $79.4 \mathrm{kcal} / \mathrm{mol}(\mathrm{G} 2(\mathrm{MP} 2))$. $\mathrm{CCSD}(\mathrm{T})$ is the single-reference method, which is in closest agreement with MCQDPT, although the difference between these two values is greater than in the case of the C-NH${ }_{2}$ BDEs of aminoethylene. The B3LYP value of $68.8 \mathrm{kcal} / \mathrm{mol}$ is in good agreement with $\operatorname{CCSD}(\mathrm{T})(70.9 \mathrm{kcal} / \mathrm{mol})$, although the B3P86 prediction of $74.5 \mathrm{kcal} / \mathrm{mol}[15]$ is closest to MP2 $(77.7 \mathrm{kcal} / \mathrm{mol})$. Similar observations apply to the predicted $\mathrm{C}-\mathrm{NO}_{2}$ bond energies in 1-amino-2-nitroethylene, which range from 69.3 (MCQDPT) to $88.8 \mathrm{kcal} / \mathrm{mol}$ (G2(MP2)). CCSD(T) (78.2 $\mathrm{kcal} / \mathrm{mol}$ ) is the single-reference method in closest agreement with the MCQDPT result and also is in good agreement with the B3LYP value $(81.4 \mathrm{kcal} / \mathrm{mol})$. MCQDPT also predicts the smallest $\mathrm{C}-\mathrm{NO}_{2}$ BDE for 1-amino-2-nitroethylene (69 $\mathrm{kcal} / \mathrm{mol}$ ). Although the $\mathrm{C}-\mathrm{NO}_{2} \mathrm{BDE}$ calculations are not yet complete, it appears that (a) G2(MP2) and MP2 consistently give the highest $\mathrm{C}_{-} \mathrm{NO}_{2}$ BDEs and (b) the single reference method in best agreement with MCQDPT is CCSD(T).

\subsubsection{High-Energy Density Materials}

A recent major focus centers around a series of proposed monopropellants.16], such

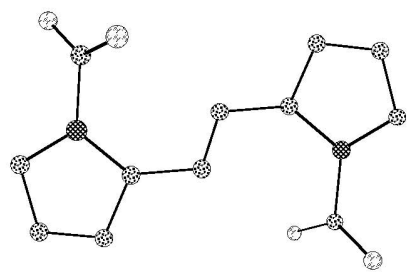

as:

$I_{s p}$, which is a measure of the energy released by the reaction of the fuel divided by the mass of the products, is of primary interest:

$$
I_{\mathrm{sp}} \propto[\Delta \mathrm{H} / \mathrm{m}]^{1 / 2}
$$

Using a combination of isodesmic reactions and the G2 model, the heat of formation for this species is predicted to be $456.8 \mathrm{kcal} / \mathrm{mol}$. This translates to an $I_{\mathrm{sp}}$ of $240 \mathrm{sec}$, compared with $230 \mathrm{sec}$ for hydrazine, thus being a very promising fuel. However, it is important to consider the stability of such high-energy species to various reactions, before asserting their viability as fuels. One possible reaction is the loss of molecular nitrogen to form the smaller species shown below:

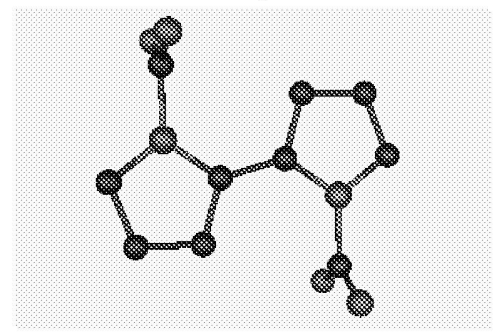

Using our highly scalable second order perturbation theory code with a large basis set, we have found that this decomposition process is exothermic by $60 \mathrm{kcal} / \mathrm{mol}$. It 
may be that the barrier for decomposition is large, in which case the species may still be viable. The search for the transition state for this reaction is in progress.

Recently the first new all-nitrogen compound in nearly a century $\mathrm{N}_{5}^{+}[17]$ was synthesized. The counterion for this species was $\mathrm{AsF}_{6}$, the result being a rather unstable solid. In order to understand the potential energy surface for this species, we performed MP2 calculations on the various stationary points. The first step in this process was to separate the two ions by a large distance and optimize the geometry. The result was an ion pair $\left[\mathrm{AsF}_{6}{ }^{-}\right]\left[\mathrm{N}_{5}^{+}\right]$complex that is, not surprisingly, $98 \mathrm{kcal} / \mathrm{mol}$ below the separated ions. What was somewhat surprising is that this complex is 47 $\mathrm{kcal} / \mathrm{mol}$ below the neutral species that is formed when one $\mathrm{F}$ ion is transferred to $\mathrm{N}_{5}^{+}$ to form neutral $\mathrm{AsF}_{5}+\mathrm{FN}_{5}$. The former is a well-known species, but the latter has not previously been reported. $\mathrm{FN}_{5}$ has several isomers, whose relative energies and the barriers separating them were determined using large basis set $\operatorname{CCSD}(\mathrm{T})$ calculations. The lowest lying isomers were found to have small energy barriers $(<10 \mathrm{kcal} / \mathrm{mol})$ separating them from the much more stable decomposition products $\mathrm{FN}_{3}+\mathrm{N}_{2}$. The exothermicity of this decomposition is more than $50 \mathrm{kcal} / \mathrm{mol}$, so the products $\mathrm{AsF}_{5}+$ $\mathrm{FN}_{3}+\mathrm{N}_{2}$ are slightly lower in energy than the original ion pair $\left[\mathrm{AsF}_{6}\right]\left[\mathrm{N}_{5}^{+}\right]$. Since $\mathrm{FN}_{3}$ is itself an energetic species with a relatively small barrier to further decomposition, it is therefore not surprising that the original species made by Christe et al. is unstable.

\subsubsection{Quantum Molecular Dynamics (MD) Simulations of Low Temperature HEDM}

Traditional MD algorithms can be extended to incorporate equilibrium quantum mechanical effects through the use of discretized Feynman path integrals[18]. The resulting algorithm, path integral MD (PIMD), is highly amenable to parallelization because each physical particle becomes rigorously mapped onto a collection of classical-like quasiparticles at different values of imaginary time, each connected to its neighbor by a harmonic-like force. The discretized PIMD algorithm can be loadbalanced across computational nodes because the quasiparticles with the same imaginary time index experience identical forces. The inter-node communications are minimal since there are only nearest-neighbor harmonic forces to compute. The PIMD algorithm, no matter how complex the physical system, can always be made to achieve excellent scalability and thus high performance. Moreover, classical MD techniques such as constant temperature and pressure algorithms can be readily incorporated into PIMD. The algorithm is even amenable to a two-tier level of parallelism, the first being over the imaginary time slices (quasiparticles), the second over the force loop for the interparticle interactions in large (thousand or even million) particle simulations.

In an important theoretical advance[19,20,21,22], PIMD methods have been extended to include quantum dynamical effects (i.e., not just equilibrium properties). This computational approach is called "Centroid Molecular Dynamics" (CMD). It incorporates the dominant quantum dynamical effects of a many-body system into a classical-like MD framework, thus significantly extending the range and applicability of MD methods. The basic result of CMD[19-22] is that the dynamical correlations of quantum particles in a general many-body system can be accurately computed by running classical-like trajectories on an effective potential which includes the effects of quantum zero point energy and tunneling. The task of integrating the CMD equations is not trivial since the effective potential is a quantum potential of mean force, requiring an "on the fly" dynamical averaging at each timestep. A number of powerful algorithms for solving the CMD equation have been developed[22]. The best CMD algorithm so far is the "hyperparallel" CMD approach[23] using an 
adiabatic algorithm[22] in the first tier of parallelism to determine the effective potential; a second tier of parallelism can be introduced in which, for each of the averaging slaves, the MD steps are sent to additional nodes for each discretized path integral imaginary time slice[23]. A third tier of parallelism for very large systems for each imaginary time slice, the classical force loop is split over several nodes. The scaling of the hyperparallel CMD code on the IBM SP and SGI O2000, as well as a Pentium Linux cluster, is excellent (Fig. 1). The parallelization of the long rage forces (Ewald summation) was also achieved.

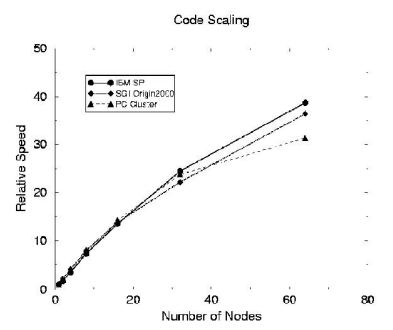

Fig. 1. Scaling of CMD

The PIMD and CMD algorithms are of general interest in all areas of condensed matter computer simulation. However, they are particularly important for the HEDM program in the computational study of, e.g., solid hydrogen, helium, or nitrogen matrices which contain isolated reactive species such as lithium, boron, aluminum, hydrogen atoms, organic radicals, etc. Such matrices are high priority targets as possible HEDMs for Air Force space propulsion purposes. At the temperatures appropriate to the condensed phases of hydrogen or helium, the host matrix molecules will exhibit large quantum mechanical effects, but the treatment of these effects by a direct attack on the time-dependent Schrödinger equation is impossible. The key issue is the stability of the HEDM (i.e., the ability to trap the energetic impurities for some period of time and thus impede their diffusion and eventual recombination). Both the structural and dynamical aspects of the composite condensed phase HEDM systems are of interest. For the equilibrium structural studies, PIMD methods are adequate. For the dynamical studies, the CMD approach must be employed to directly simulate the diffusive and recombination steps of the guest atoms in the solid host. The longterm goal of this research is to develop general computational methods to rapidly and efficiently characterize proposed high energy density materials.

The structure and stability of atomic impurities trapped in solid para-hydrogen have been studied by employing large scale PIMD and CMD simulations at $4 \mathrm{~K}$ and zero external pressure. Starting from pure solid hydrogen, doped systems were prepared by substituting impurity atoms for hydrogen molecules at substitutional defect sites. The structural and thermodynamic properties of the resulting HEDM were then calculated. In the case of atomic boron impurities, the impurity atom interacts anisotropically with the matrix para-hydrogen molecules because of its singly filled $2 p$ orbital. To assess the effect of the electronic anisotropy in the potential, a comparison has been made between simulations in which the orientationdependent (anisotropic) and orientation-averaged (spherical) $\mathrm{B}-\mathrm{H}_{2}$ potentials were used. In collaboration with the Alexander group[24], three matrices were investigated: (a) one with a single $\mathrm{B}$ atom site-substituted for a $p-\mathrm{H}_{2}$ molecule, (b) one with a similar site-substituted matrix having a nearest-neighbor vacancy, (c) and one with a periodic "super cell" simulation box having two hydrogen bulk slab regions in order to study a B impurity near the $p-\mathrm{H}_{2}$ surface. 
It was found that significant "second-order" distortions of the lattice occur to permit an energetically favorable orientation of the $2 p$ orbital, even in the absence of a neighboring vacancy. Especially when the B impurity is located near the surface, the spherically averaged potential provides a noticeably different description from the case of the anisotropic potential. These quantum MD simulations reveal the large structural differences that can be found in the lattice resulting from the anisotropic boron-hydrogen interaction potential. The implications of this behavior will be explored in future research.

\subsection{POSS Compounds}

There is great interest in POSS compounds because of their resistance to extreme environments and the ease with which they are synthesized. Since almost nothing is known experimentally about the mechanism by which they are formed, we have embarked on a long term project to determine the possible competing mechanisms as a function of catalyst, solvent, and substituents (e.g., in[25]). The synthesis begins with hydrolysis of $\mathrm{RSiX}_{3}$ to $\mathrm{RSi}(\mathrm{OH})_{3}$, followed by condensation to the siloxane $\mathrm{RSi}(\mathrm{OH})_{2}-\mathrm{O}-\mathrm{Si}(\mathrm{OH})_{2} \mathrm{R}$. Subsequent condensations lead to the 3D cage compounds. We have shown that the initial hydrolsysis and condensation steps all have very highenergy barriers in the gas phase, but are being reduced to nearly zero by the presence of one water molecule added to represent the solvent. Thus, all steps leading to the initial disiloxanes and to the ring compounds $D_{3}$ and $D_{4}$ occur with net energy requirements of less than $10 \mathrm{kcal} / \mathrm{mol}$. In this paper we describe current efforts to (a) determine substituent effects on these barriers, (b) determine the effects of adding additional waters, and (c) to compare the properties of the 3D POSS compounds with their $\mathrm{Ti}$ analogs. The substituents studied include $\mathrm{R}=\mathrm{H}$, methyl, $\mathrm{t}$-butyl and phenyl, and $\mathrm{X}=\mathrm{Cl}, \mathrm{OCH}_{3}$. There is experimental interest in synthesizing incompletely condensed POSS. Because the reaction is very fast, it always goes to completion. The interest in substituent effects lies in attempting to slow down the reaction, possibly using bulky R groups. There is industrial interest in the possibility that Ti POSS may be another class of materials with desirable properties. Hammes-Schiffer et al.[26] have been developing methodologies for the simulation of hydrogen transfer reactions, and most recently a new molecular orbital method for the simultaneous calculation of electronic and hydrogen vibrational wavefunctions, now incorporated into GAMESS. This method may be used to obtain minimum energy reaction paths and direct dynamics trajectories of hydrogen transfer reactions, with the advantage that nuclear quantum effects such as zero point energy and hydrogen tunneling are incorporated during the generation of the reaction paths and trajectories, rather than subsequently included as corrections. This approach will be combined with a mixed quantum/classical surface hopping method to study the quantum dynamics of hydrogen transfer reactions with an $a b$ initio potential energy surface obtained "on the fly," and used to investigate hydrogen transfer reactions in the hydrolysis and condensation steps required for the synthesis of POSS, in order to determine the influence of different trihalosilane reactants on the rates and yields of these steps and aid in the efficient synthesis of POSS.

\subsection{Nonlinear Absorbing Materials}

We have previously shown that density functional theory and the time-dependent density functional response theory (TDDFT) (B3LYP) accurately predict the structures and spectra of porphin and the larger tetraphenylporphyrin and its $\beta$ - 
octahalogenated derivatives,[27],28] thus enabling their assessment as reverse saturable absorbing materials For $\mathrm{PH}_{2}$, the photoinduced tautomerization process has also been investigated[29] in order to discern between previously proposed discordant mechanisms. Recently we carried out calculations[30] for tetrabenzoporphyrin (ZnTBP) and phthalocyanine ( $\mathrm{ZnPc})$ (Fig. 2).

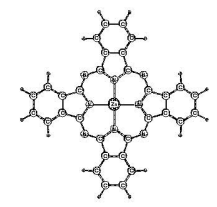

Fig. 2. Zinc phthalocyanine

Our calculations support the multiple bands in the B and higher energy regions of the $\mathrm{ZnPc}$ and $\mathrm{ZnTBP}$ spectra that have been determined from experiment. Despite considerable experimental and theoretical work on $\mathrm{ZnPc}$ and $\mathrm{ZnTBP}$, their ground state electronic structures and UV spectra are not well understood. Previous theoretical studies provided only a qualitative understanding in the low-energy region, and none for the higher energy bands. We have elucidated significant shifts in Gouterman's four orbitals of $\mathrm{ZnP}$ upon tetraazasubstitution and tetrabenzoannulation (detailed in[30]). The benzo groups destabilize the $1 \mathrm{a}_{1 \mathrm{u}}$ orbital in $\mathrm{ZnP}$, but they do not show a significant deviation from the four-orbital model for the interpretation of the $\mathrm{Q}$ and $B$ bands of ZnTBP. However, tetraazasubstitions lower considerably the $4 \mathrm{a}_{2 u}$ orbital (HOMO-1) in ZnTBP. Also, it is shown that the near degeneracy of the HOMO and HOMO-1 in $\mathrm{ZnP}$ that provides the basis for Gouterman's four orbital model does not hold for $\mathrm{ZnPc}$. In $\mathrm{ZnPc}$, the near degeneracy of the HOMO-1 $\left(4 \mathrm{a}_{2 \mathrm{u}}\right)$ with other occupied orbitals gives rise to a complex structure of the higher energy regions of the spectrum that were not reproduced by simple MO methods. The $4 \mathrm{a}_{2 u}$ MO has significant $\pi$ contributions from the benzo rings in $\mathrm{ZnPc}$ but not in $\mathrm{ZnTBP}$. The Q and B bands are predicted to appear at 2.18 and $3.28 \mathrm{eV}$ for ZnTBP, respectively, in excellent agreement with the maxima located at 2.02 and $3.18 \mathrm{eV}$ as established from the absorption spectrum in a supersonic jet expansion, with similar values $(2.02 \& 3.10 \mathrm{eV})$ obtained from the absorption spectrum in an Ar matrix. Tetraazasubstitutions are predicted to redshift the Q band of ZnTBP by about $0.1 \mathrm{eV}$. The computed excitation energies in near quantitative agreement with experiment clearly provide a strong basis for the interpretation of the electronic spectra of such large molecular systems. TDDFT calculations are also being carried out for candidate two-photon absorption (TPA) materials,[31] to be compared to those of Albota et al.[32]

\subsection{Liquid Crystals}

Recent work is in progress for studying a liquid crystalline droplet to model the behavior of a liquid crystal in the bulk, with the details reported elsewhere.[33] Indeed, atomic level simulations are proven important in understanding the structureproperty relations of materials. Due to the size of the LC droplet very large-scale classical MD simulations of such systems are required, that may, become computationally prohibitive. The fast multipole method (FMM)[34], which uses a multiscale hierarchy of partitions of the volume and a divide-and-conquer strategy to compute the power series, allows all the forces to be computed to any specified 
accuracy in $O(\mathrm{~N})$ operations. FMM3D (an implementation and improvement to FMM in 3-D), which contains a variety of schemes for computing multipole translations, has been implemented into the MD program that is being developed in our group.[35]

\section{References}

1. Pople, J.A., Binkley, J.S., Seeger: R., Int. J. Quantum Chem., 10 (1976) 1; Bartlett, R.J., Silver, D.M.: Int. J. Quantum Chem., 9 (1975) 183; Dupuis, M., Chin, S., Marquez, A.: In: Relativistic and Electron Correlation Effects in Molecules, Malli, G.: Ed. Plenum Press, NY 1994; Frisch, M.J., Head-Gordon, M., Pople, J.A.: Chem. Phys. Lett. 1660 (1990) 275-280

2. Curtiss, L.A., Raghavachari, K., Pople, J.A.: J. Chem. Phys. 98 (1993) 1293

3. See, for example, Bartlett, R.J., Stanton, J.F.: In: Applications of Post-Hartree-Fock Methods: A Tutorial, Reviews of Computational Chemistry, Vol. V, Lipkowitz, K.B. and Boyd, D.B., eds., VCH Publishers, Inc. New York, 1994

4. Siegbahn, P.E., Heiberg, A., Roos, B.O., Levy, B.: Phys. Scr. 21 (1980) 232. b) Roos, B.O., Taylor, P.R., Siegbahn, P.E.: Chem. Phys. 48 (1980) 157. c) Roos, B.O.: Int. J. Quantum Chem. S14 (1980) 175

5. Nakano, H.: J. Chem. Phys. 99 (1993) 7983-7992

6. Becke, A.D.: J. Chem. Phys. 98 (1993) 5648

7. Bemm, U., Östmark, H.: Acta Cryst. C54 (1998) 1997; Östmark, H., Langlet, A., Bergman, H., Wellmar, U., Bemm, U.: Eleventh Int. Detonation Symp., 30 Aug - 4 Sep 1998, Snowmass Village, Colorado, USA

8. Krishnan, R., Binkley, J.S., Seeger, R., Pople, J.A.: J. Chem. Phys. 72 (1980) 650. (Sixcomponent Cartesian d functions used.)

9. Boatz, J.A., Sorescu, D.C., Thompson, D.L.: manuscript in preparation

10. Gordon, M.S., Schmidt, M.W., Chaban, G.M., Glaesemann, K.R., Stephens, W.J., Gonzales, C.: J. Chem. Phys. 110 (1999) 4199

11. Schmidt, M.W., Baldridge, K.K., Boatz, J.A., Elbert, S.T., Gordon, M.S., Jensen, J.J., Koseki, S., Matsunaga, N., Nguyen, K.A., Su, S., Windus, T.L., Dupuis, M., Montgomery, J.A.: J. Comput. Chem. 14 (1993) 1347.

12. Gaussian 98, Revision A.7, Frisch, M. J., Trucks, G.W., Schlegel, H.B., Scuseria, Robb, M.A., Cheeseman, J.R., Zakrzewski, V.G., Montgomery, J.A., Jr., Stratmann, R.E., Burant, J.C., Dapprich, S., Millam, J.M., Daniels, A.D., Kudin, K.N., Strain, M.C., Farkas, O., Tomasi, J., Barone, V., Cossi, M., Cammi, R., Mennucci, B., Pomelli, C., Adamo, C., Clifford, S., Ochterski, J., Petersson, G.A., Ayala, P.Y., Cui, Q., Morokuma, K., Malick, D.K., Rabuck, A.D., Raghavachari, K., Foresman, J.B., Cioslowski, J., Ortiz, J.V., Baboul, A.G., Stefanov, B.B., Liu, G., Liashenko, A., Piskorz, P., Komaromi, I., Gomperts, R., Martin, R.L., Fox, D.J., Keith, T., Al-Laham, M.A., Peng, C.Y., Nanayakkara, A., Gonzalez, C., Challacombe, M., Gill, P.M.W., Johnson, B., Chen, W., Wong, M.W., Andres, J.L., Gonzalez, C., Head-Gordon, M., Replogle, E.S., and Pople, J.A.: Gaussian, Inc., Pittsburgh PA, 1998

13. Stanton, J.F., Gauss, J., Watts, J.D., Lauderdale, W.J., and Bartlett, R.D.: Int. J. Quantum Chem., S26 (1992) 879

14. Lias, S.G., Bartmess, J.E., Liebman, J.F., Holmes, J.L., Levin, R.D., and Mallard, W.G: J. Phys. Chem. Ref. Data 17 (Suppl. 1) (1988) 1-872

15. Politzer, P., Concha, M.C., Grice, M.E., Murray, J.S., Lane, P., Habibollazadeh, D.: Theochem, 452 (1998) 75

16. Work of the late Rob Schmitt \& Jeff Botaro, SRI

17. Christe and co-workers, AFRL/PR

18. See, e.g., Berne, B.J., and Thirumalai, D.: Annu. Rev. Phys. Chem. 37 (1987) 401

19. Cao, J. and Voth, G.A.: J. Chem. Phys. 99 (1993) 10070

20. Cao, J. and Voth, G.A.: J. Chem. Phys. 100 (1994) 5106

21. Cao, J. and Voth, G.A.: J. Chem. Phys. 101 (1994) 6157 
22. Cao, J. and Voth, G.A.: J. Chem. Phys. 101 (1994) 6168

23. Calhoun, A., Pavese, M., and Voth, G.A.: Chem. Phys. Lett. 262 (1996) 415

24. Krumrine, J.R., Jang, S., Alexander, M.H., and Voth, G.A.: J. Chem. Phys. 113 (2000) 9079

25. Kudo, T., Takako, and Gordon, M.S.: J. Phys. Chem. A, 104 (2000) 4058

26. S. Hammes-Schiffer: J. Phys. Chem. A 102 (1998) 10443; Webb, S.P., Agarwal, P.K., and Hammes-Schiffer, S.: J. Phys. Chem. B 104 (2000) 888; WebbS.P., and Hammes-Schiffer, S.: J. Chem. Phys. 113 (2000) 5214

27. Nguyen, K. A., Day, P. N., and Pachter, R.: J. Chem. Phys,. 110 (1999) 9135; Nguyen, K. A., Day, P. N., and Pachter, R.: J. Phys. Chem. A, 103 (1999) 7378

28. Nguyen, K. A., Day, P. N., and Pachter, R.: J. Phys. Chem., 104 (2000) 4755

29. Nguyen, K. A. and Pachter, R., J. Phys. Chem., 104 (2000) 4549

30. Nguyen, K.A., and R. Pachter, J. Chem. Phys., manuscript in preparation

31. Day, P.N., and Pachter, R., work in progress

32. Albota, M., Beljonne, D., Bredas, J.L., Ehrlich, J.E., Fu, J.Y., Heikal, A.A., Hess, S.E., Kogej, T., Levin, M.D., Marder, S.R., McCord-Maughon, D., Perry, J.W., Rockel, H., Rumi, M., Subramaniam, G., Webb, W.W., Wu, X.L., and Xu, C.: Science 281 (1998) 1653

33. Wang, Z., Patnaik, S., and Pachter, R.: Computational and Theoretical Polymer Science, to appear, 2001

34. Greengard, L., and Rokhlin, V.: J. Comp. Phys., 73 (1987) 325

35. Wang, Z., Lupo, J.A., McKenney, A., and Pachter, R.: Proceedings of SC99 (1999) 